Bulletin of the Biometric Soc. of Japan

Vol.12, No.1 \& No.2(1991). pp.57-65

\title{
Approximate and Exact Methods for Computing the Confidence Limits for the Common Odds Ratio in a Series of $2 \times 2$ Tables
}

\author{
Hirofumi Takagi \\ The Institute of Statistical Mathematics \\ 4-6-7 Minami-Azabu, Minato-ku, Tokyo 106, Japan
}

\begin{abstract}
Summary
For computing the confidence limits of the common odds ratio in $K 2 \times 2$ tables, Monte Carlo experiments compared respective performances of the original exact method (Gart, 1970, and Zelen, 1971), the mid-P (Lancaster, 1961) modification methods, the ln-method using the conditional maximum likelihood estimate and its variance, or using the MantelHaenszel estimate and its asymptotic variance proposed by Robins et al (1986), and the Sato's method (1990) with and without a continuity correction. Approximate methods gave the accurate and symmetric lower and upper percentages when the true common odds ratio was unity, while upper percentages became liberal but lower percentages conservative, reversely, as the true common odds ratio increased, especially in matched designs. The original exact method and the Sato's method with the correction gave conservative results. The mid-P exact method gave the most accurate, symmetric and nearest lower and upper percentages to the nominal size, regardless of the true common odds ratio, the sample size, and the number of strata.
\end{abstract}

\section{Introduction}

In the epidemiologic studies, especially in case-control studies, the data are often stratified into a series of $2 \times 2$ tables to elucidate a dichotomous risk factor without the influences of the confounding variables. We usually compute the estimate and confidence limits for the common odds ratio, $\psi$, in $K \cdot 2 \times 2$ independent contingency tables. Several asymptotic methods have been proposed to this problem.

The Mantel-Haenszel (1959) estimate, $\hat{\psi}_{M H}$, is ordinarily used to estimate $\psi$. To construct the confidence limits for $\psi$, the asymptotic variance of the Mantel-Haenszel estimator was given by Hauck (1979) based on "large-stratum" sets wherein the number of tables remained fixed but individual cell sizes increased without bound. Breslow (1981) proposed one variance for "sparse-data" settings where the cell sizes remained bounded but the number of tables increased. Robins et al (1986) provided a good asymptotic variance for both sparse-data and large-stratum settings. We may construct an approximate

Key words: Case-control study; Common odds ratio; Conditional distribution; Epidemiology; Exact method; Mantel-Haenszel estimator. 
confidence interval by the "ln-method" that is based on a normal distribution with a mean of $\log \hat{\psi}_{M H}$ and its asymptotic variance.

Another approximate methods are derived from the test statistics such as the extended Cornfield method by Gart $(1970,1971)$ that performs well for large-stratum sets but poorly for sparse-data sets, while Sato (1990) proposed a good method for both sparsedata and large-stratum settings based on the estimating function for the common odds ratio.

However, we can also construct an exact confidence interval based on the conditional distribution (Gart, 1970, and Zelen, 1971), which guarantees the desired coverage probabilty. Though the computational complexity exists in estimating the exact confidence limits, Mehta et al (1985) provided the network algorithm and also Takagi (1990) developed a simple recursive algorithm to overcome the time consumption even for sparse-data settings.

To give the answer what method should be recommended, in this paper, we compare the performances of approximate methods based on the asymptotic distribution of the logarithm of the Mantel-Haenszel estimator and the conditional maximum likelihood estimator, and the estimating function with those of the original exact and mid-P methods through several Monte Carlo experiments.

\section{Exact Confidence Limits}

Consider $K 2 \times 2$ tables with pairs of independent binomial observations $\left(x_{k}, y_{k}\right)$ with denominators $\left(n_{k}, m_{k}\right)$ and success probabilities $\left(p_{1 k}, p_{0 k}\right)$ for $k=1, \ldots, K$. In casecontrol studies, $x_{k}$ and $y_{k}$ persons are exposed in $n_{k}$ cases and $m_{k}$ controls respectively in the $k$ th stratum. To measure the disease-exposure association, the odds ratio $\psi_{k}=$ $\left(p_{1 k} q_{0 k}\right) /\left(p_{0 k} q_{1 k}\right)$ is always available, where $q_{1 k}=1-p_{1 k}$ and $q_{0 k}=1-p_{0 k}$ in the $k$ th table. Usually assumed $\psi_{k}=\psi$ for all $k$, where $\psi$ is the common odds ratio. The MantelHaenszel estimate is obtained as $\hat{\psi}_{M H}=\sum_{k}\left[x_{k}\left(m_{k}-y_{k}\right) / N_{k}\right] / \sum_{k}\left[y_{k}\left(n_{k}-x_{k}\right) / N_{k}\right]$, where $N_{k}=n_{k}+m_{k}$.

The "exact" estimator and confidence limits for $\psi$ are derived from the conditional distribution (Gart, 1970, and Zelen, 1971). Given the table marginals $n_{k}, m_{k}$ and $t_{k}=$ $x_{k}+y_{k}$, the conditional distribution is the noncentral hypergeometric distribution for $x_{k}$,

$$
f\left(x_{k} \mid t_{k}, \psi\right)=\frac{\left(\begin{array}{c}
n_{k} \\
x_{k}
\end{array}\right)\left(\begin{array}{c}
m_{k} \\
t_{k}-x_{k}
\end{array}\right) \psi^{x_{k}}}{\sum_{r_{k}}\left(\begin{array}{c}
n_{k} \\
r_{k}
\end{array}\right)\left(\begin{array}{c}
m_{k} \\
t_{k}-r_{k}
\end{array}\right) \psi^{r_{k}}}
$$

where $l_{k}=\max \left(0, t_{k}-m_{k}\right) \leq r_{k} \leq \min \left(n_{k}, t_{k}\right)=u_{k}$.

The conditional maximum likelihood estimate (CMLE) $\hat{\psi}_{c}$ can be obtained by the following equation with an iterative method.

$$
\sum_{k=1}^{K} x_{k}=\sum_{k=1}^{K} \sum_{r_{k}=l_{k}}^{u_{k}} r_{k} f\left(r_{k} \mid t_{k}, \hat{\psi}_{c}\right) .
$$


Recently, according to the developmerts of computer and algorithm for the calculation, the CMLE can be easily computed without time spending even by 16 bit micro-computer (Satten and Kupper, 1990, and Takagi, 1990).

Let $L=\sum_{k} l_{k}$ and $U=\sum_{k} u_{k}$, and by using (1), the conditional distribution of $s=$ $\sum_{k} x_{k}$ is given (Zelen, 1971) by

$$
g(s \mid \psi)=\frac{C_{s} \psi^{s}}{\sum_{r=L}^{U} C_{r} \psi^{r}}
$$

where

$$
C_{s}=\sum_{R(s)} \prod_{k=1}^{K}\left(\begin{array}{c}
n_{k} \\
r_{k}
\end{array}\right)\left(\begin{array}{c}
m_{k} \\
t_{k}-r_{k}
\end{array}\right),
$$

and $R(s)$ is the set of all $K$-fold partitions of all $\sum_{k} r_{k}=s$.

Hence, exact $100(1-\alpha) \%$ confidence limits for $\psi$ are given by $\left\{\hat{\psi}_{L}, \hat{\psi}_{U}\right\}$ such that

$$
\sum_{z=s}^{U} g\left(z \mid \hat{\psi}_{L}\right)=\frac{\alpha}{2}
$$

and

$$
\sum_{z=L}^{s} g\left(z \mid \hat{\psi}_{U}\right)=\frac{\alpha}{2}
$$

Note that $\hat{\psi}_{L}=0$ if $s=L$, and $\hat{\psi}_{U}=\infty$ if $s=U$.

To estimate exact confidence limits, we may use the network algorithm by Mehta et al (1985) or the simple recursive algorithm by Takagi (1990).

Since equations (4) and (5) provide a conservative confidence interval as Mehta et al (1985) pointed out, if liberal confidence limits are preferable, we may modify the original exact method; i.e., the "mid-P" method (Lancaster, 1961, Rothman, 1986, and Vollset and Hirji, 1991) for estimating equations as follows;

$$
\frac{1}{2} g\left(s \mid \hat{\psi}_{L}\right)+\sum_{z=s+1}^{U} g\left(z \mid \hat{\psi}_{L}\right)=\frac{\alpha}{2}
$$

and

$$
\frac{1}{2} g\left(s \mid \hat{\psi}_{U}\right)+\sum_{z=L}^{s-1} g\left(z \mid \hat{\psi}_{U}\right)=\frac{\alpha}{2} .
$$

\section{Approximate Confidence Limits}

To construct the confidence limits for $\psi$, the asymptotic variance of the Mantel-Haenszel estimator was given by Hauck (1979) based on large-stratum sets. Breslow (1981) proposed one variance for sparse-data settings. Robins et al (1986) provided a good asymptotic variance, $V_{R}$, for both sparse-data and large-stratum settings as follows;

$$
V_{R}=\frac{1}{2} \sum_{k=1}^{K}\left[\frac{P_{k} R_{k}}{R_{+}^{2}}+\frac{Q_{k} R_{k}+P_{k} S_{k}}{R_{+} S_{+}}+\frac{Q_{k} S_{k}}{S_{+}^{2}}\right],
$$


where $R_{k}=x_{k}\left(m_{k}-y_{k}\right) / N_{k}, S_{k}=y_{k}\left(n_{k}-x_{k}\right) / N_{k}, P_{k}=\left(x_{k}+m_{k}-y_{k}\right) / N_{k}, Q_{k}=$ $\left(y_{k}+n_{k}-x_{k}\right) / N_{k}, R_{+}=\sum_{k} R_{k}$, and $S_{+}=\sum_{k} S_{k}$.

We may construct an approximate confidence interval by the ln-method based on the asymptotic distribution of the logarithm of the Mantel-Haenszel estimator. The approximate $(1-\alpha)$ confidence limits are given by

$$
\left(\psi_{l}, \psi_{U}\right)=\hat{\psi}_{M H} \exp \left[ \pm \chi_{\alpha} \sqrt{V\left(\log \hat{\psi}_{M H}\right)}\right],
$$

where $\chi_{\alpha}^{2}$ is the upper $\alpha$ point of the chi-square distribution with one degree of freedom.

For constructing the confidence interval by the In-method, We may use the CMLE $\psi_{c}$ given by (2) and the asymptotic variance of the logarithm of $\psi_{c}$,

$$
V_{c}=\left[\sum_{k=1}^{K}\left\{G_{k}\left(x_{k}^{2} \mid t_{k}, \hat{\psi}_{c}\right)-G_{k}^{2}\left(x_{k} \mid t_{k}, \hat{\psi}_{c}\right)\right\}\right]^{-1},
$$

where

$$
G_{k}\left(x_{k}^{a} \mid t_{k}, \hat{\psi}_{c}\right)=\frac{\sum_{r_{k}} r_{k}^{a}\left(\begin{array}{c}
n_{k} \\
r_{k}
\end{array}\right)\left(\begin{array}{c}
m_{k} \\
t_{k}-r_{k}
\end{array}\right) \hat{\psi}_{c}^{r_{k}}}{\sum_{r_{k}}\left(\begin{array}{c}
n_{k} \\
r_{k}
\end{array}\right)\left(\begin{array}{c}
m_{k} \\
t_{k}-r_{k}
\end{array}\right) \hat{\psi}_{c}^{r_{k}}} .
$$

Another approximate methods are derived from the test statistics such as the extended Cornfield method by Gart $(1970,1971)$ that performs well for large-stratum sets but poorly for sparse-data sets, while Sato (1990) proposed a good method for both sparsedata and large-stratum settings based on the estimating function, $R_{k}-\psi S_{k}$. The approximate $(1-\alpha)$ confidence limits $\left(\psi_{L}, \psi_{U}\right)$ are the solutions of the quadratic equation

$$
\frac{\left(\left|R_{+}-\psi S_{+}\right|-c\right)^{2}}{\psi W}=\chi_{a}^{2}
$$

where $W=\sum_{k}\left[\left(Q_{k}+N_{k}^{-1}\right) R_{k}+\left(P_{k}+N_{k}^{-1}\right) S_{k}\right]$, and $c=(1+\psi) / 4$ when a continuity correction is needed, or $c=0$, otherwise. Solving (10), Sato(1990) showed the upper and lower limits

$$
\left(\psi_{L}, \psi_{U}\right)=\frac{2 R_{+} S_{+}+\chi_{\alpha}^{2} W \pm \sqrt{\left(4 R_{+} S_{+}+\chi_{\alpha}^{2} W\right) \chi_{\alpha}^{2} W}}{2 S_{+}^{2}},
$$

without a continuity correction, and when it is needed, change $R_{+}$and $S_{+}$to $R_{+}-\frac{1}{4}$ and $S_{+}+\frac{1}{4}$ for calculating $\psi_{L}$, and to $R_{+}+\frac{1}{4}$ and $S_{+}-\frac{1}{4}$ for calculating $\psi_{U}$.

\section{Monte Carlo Experiments}

Several Monte Carlo experiments were conducted to compare the performances of the original exact and mid-P methods, equations (4) to (8), with those of the ln-method using the logarithm of the Mantel-Haenszel estimate $\hat{\psi}_{M H}$ and its asymptotic variance $V_{R}$, the logarithm of the CMLE $\hat{\psi}_{c}$ and its variance $V_{c}$, and also with those of Sato's equation (12) with and without the continuity correction. 
Table 1

Percentage of trials in which $\psi$ fell bellow the lower or above the upper bound of the $95 \%$ confidence interval, $\psi=1.0$.

\begin{tabular}{|c|c|c|c|c|c|c|c|c|c|c|c|c|c|c|}
\hline \multirow{4}{*}{$n$} & \multirow{4}{*}{$m$} & \multirow{4}{*}{$K$} & \multirow{2}{*}{\multicolumn{4}{|c|}{ Exact method }} & \multirow{2}{*}{\multicolumn{4}{|c|}{ ln-method }} & \multicolumn{4}{|c|}{ Sato } \\
\hline & & & & & & & & & & & \multirow{2}{*}{\multicolumn{2}{|c|}{$\begin{array}{c}\text { Without } \\
\text { corr. }\end{array}$}} & \multirow{2}{*}{\multicolumn{2}{|c|}{$\begin{array}{l}\text { With } \\
\text { corr. }\end{array}$}} \\
\hline & & & \multicolumn{2}{|c|}{ Original } & \multicolumn{2}{|c|}{ mid-P } & \multicolumn{2}{|c|}{$\bar{\psi}_{c}, V_{c}$} & \multicolumn{2}{|c|}{$\hat{\psi}_{M H}, V_{R}$} & & & & \\
\hline & & & $\mathrm{L}$ & $\bar{U}$ & $\mathrm{~L}$ & & $\mathrm{~L}$ & $\bar{U}$ & $\mathrm{~L}$ & $\overline{\mathrm{U}}$ & $\mathrm{L}$ & $\bar{U}$ & $\mathrm{~L}$ & \\
\hline 1 & 1 & 200 & 2.0 & 2.0 & 2.5 & 2.5 & 2.4 & 2.4 & 2.4 & 2.4 & 2.5 & 2.5 & 1.9 & 1.9 \\
\hline & & 100 & 1.8 & 1.8 & 2.5 & 2.6 & 2.2 & 2.3 & 2.2 & 2.3 & 2.5 & 2.6 & .7 & 1.8 \\
\hline 1 & 2 & 100 & 1.8 & 1.9 & 2.4 & 2.4 & 2.3 & 2.4 & 2.3 & 2.4 & 2.4 & 2.5 & 1.8 & 1.8 \\
\hline & & 50 & 1.7 & 1.7 & 2.4 & 2.4 & 2.3 & 2.4 & 2.3 & 2.3 & 2.5 & 2.6 & 1.6 & 1.6 \\
\hline \multirow[t]{2}{*}{1} & 4 & 50 & 1.7 & 1.8 & 2.4 & 2.5 & 2.3 & 2.5 & 2.2 & 2.5 & 2.4 & 2.6 & 1.6 & 1.8 \\
\hline & & ת5 & 1.5 & 1.5 & 2.4 & 2.3 & 2.2 & 2.3 & 2.1 & 2.2 & 2.5 & 2.5 & 1.4 & 1.4 \\
\hline \multirow[t]{2}{*}{1} & 8 & 50 & 1.8 & 1.7 & 2.4 & 2.4 & 2.3 & 2.4 & 2.3 & 2.4 & 2.4 & 2.5 & 1.7 & 1.7 \\
\hline & & 25 & 1.4 & 1.5 & 2.3 & 2.3 & 2.1 & 2.3 & 2.0 & 2.3 & 2.4 & 2.6 & 1.3 & 1.5 \\
\hline \multirow[t]{2}{*}{5} & 5 & 20 & 1.7 & 1.7 & 2.4 & 2.3 & 2.4 & 2.3 & 2.4 & 2.3 & 2.6 & 2.5 & 1.8 & 1.7 \\
\hline & & & 1.4 & 1.5 & & 2.3 & 2.3 & 2.3 & 2.3 & 2.3 & 2.6 & 2.6 & 1.4 & 1. \\
\hline \multirow{3}{*}{15} & 15 & 20 & 2.1 & 2.0 & 2.5 & 2.5 & 2.5 & 2.5 & 2.5 & 2.5 & 2.6 & 2.5 & 2.1 & 2.1 \\
\hline & & 10 & 1.9 & 1.8 & 2.5 & 2.4 & 2.5 & 2.4 & 2.5 & 2.4 & 2.6 & 2.5 & 1.9 & 1.8 \\
\hline & & & 1.6 & 1.6 & 2.3 & 2.4 & 2.3 & 2.4 & 2.4 & 2.4 & 2.6 & 2.7 & 1.6 & 1.6 \\
\hline \multirow[t]{3}{*}{30} & 30 & 20 & 2.1 & 2.1 & 2.5 & 2.4 & 2.5 & 2.4 & 2.5 & 2.4 & 2.5 & 2.5 & 21 & 2.1 \\
\hline & & 10 & 1.9 & 2.0 & 2.4 & 2.5 & 2.4 & 2.5 & 2.4 & 2.5 & 2.5 & 2.5 & 1.9 & 2.0 \\
\hline & & & 1.8 & 1.7 & 2.4 & 2.3 & 2.4 & 2.4 & 2.5 & 2.4 & 2.6 & 2.5 & 1.9 & 1.8 \\
\hline \multirow[t]{2}{*}{5} & 10 & 20 & & 1.8 & 2.4 & 2.4 & 2.4 & 2.4 & 2.4 & 2.4 & 2.5 & 2.5 & 1.8 & 1.8 \\
\hline & & 10 & 1.6 & 1.6 & 2.3 & 2.4 & 2.3 & 2.4 & 2.3 & 2.4 & 2.6 & 2.6 & 1.6 & 1. \\
\hline \multirow[t]{3}{*}{15} & 30 & 20 & 2.0 & 2.1 & 2.4 & 2.6 & 2.4 & 2.5 & 2.4 & & 2.5 & 2.6 & & \\
\hline & & 10 & 1.9 & 1.9 & 2.5 & 2.4 & 2.5 & 2.4 & 2.5 & 2.4 & 2.6 & 2.5 & 1.9 & 1.9 \\
\hline & & & 1.7 & 1.7 & & 2.4 & 2.4 & 2.4 & 2.4 & 2.5 & 2.6 & 2.6 & & 1.8 \\
\hline \multirow{2}{*}{30} & 60 & 10 & 2.2 & 2.0 & 2.5 & 2.4 & 2.5 & 2.4 & 2.6 & 2.4 & 2.6 & 2.4 & 2.2 & 2.0 \\
\hline & & & 1.9 & 1.9 & 2.4 & 2.5 & 2.4 & 2.5 & 2.4 & 2.5 & 2.5 & 2.6 & 1.9 & 1.9 \\
\hline
\end{tabular}

$\mathrm{L}$ and $\mathrm{U}$ stand for Lower and Upper noncoverage rates, respectively, and corr. for the continuity correction.

We performed several Monte Carlo experiments, using an experiment protocol nearly identical to those reported in Sato (1990), namely $p_{1 k}=.3+.5(k-1) /(K-1)$ for $K \in\{200,100,50,25\}, k=1, \ldots, K$, and $n_{k}=1$ and $m_{k}=1,2,4,8$ in matched designs. The experiments in balanced designs used $p_{1 k}=.05+.8(k-1) /(K-1)$ for $K \in\{20,10,5\}, k=1, \ldots, K, n_{k}=m_{k}=5,15,30$, and experiments in inbalanced designs used the same levels of $p_{1 K}, K$ and $n_{k}$ except $m_{k}=2 n_{k}=10,30,60$. All experiments used $\psi=1,3.5,6.5$, and 10000 trials per experiment in order to estimate the actual noncoverage rate of nominal $95 \%$ confidence interval.

Binomial random variables were generated using the random number generator by a physical process on a HITAC-M682H in the Institute of Statistical Mathematics. When $R_{+}=0$ or $S_{+}=0$ in a given trial, we ignored the trial for comparability because $V_{R}$ is undefined.

For each trial we computed six nominal $95 \%$ confidence limits $\left(\psi_{L}, \psi_{U}\right)$, and cumulated the number of times that the true common odds ratio fell below the lower $\left(\psi<\psi_{L}\right)$ or above the upper $\left(\psi>\psi_{U}\right)$ limit. 
Table 2

Percentage of trials in which $\psi$ fell bellow the lower or above the upper bound of the $95 \%$ confidence interval, $\psi=3.5$.

\begin{tabular}{|c|c|c|c|c|c|c|c|c|c|c|c|c|c|c|}
\hline \multirow{4}{*}{$n$} & \multirow{4}{*}{$m$} & \multirow{4}{*}{$K$} & \multirow{2}{*}{\multicolumn{4}{|c|}{ Exact method }} & \multirow{2}{*}{\multicolumn{4}{|c|}{ ln-method }} & \multicolumn{4}{|c|}{ Sato } \\
\hline & & & & & & & & & & & \multirow{2}{*}{\multicolumn{2}{|c|}{$\begin{array}{c}\text { Without } \\
\text { corr. }\end{array}$}} & \multirow{2}{*}{\multicolumn{2}{|c|}{$\begin{array}{l}\text { With } \\
\text { corr. }\end{array}$}} \\
\hline & & & \multicolumn{2}{|c|}{ Original } & \multicolumn{2}{|c|}{ mid-P } & \multicolumn{2}{|c|}{$\hat{\psi}_{c}, V_{c}$} & \multicolumn{2}{|c|}{$\bar{\psi}_{M H}, V_{R}$} & & & & \\
\hline & & & L & $\overline{\mathrm{U}}$ & $\bar{L}$ & $\overline{\mathrm{U}}$ & $\bar{L}$ & $\mathrm{U}$ & $\mathrm{L}$ & $\mathrm{U}$ & $\mathrm{L}$ & $\mathrm{U}$ & $\mathrm{L}$ & U \\
\hline 1 & 1 & 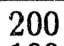 & 1.8 & 2.0 & 2.4 & 2.4 & 9 & 2.7 & 19 & 2.7 & 2.0 & 2.8 & 1.5 & 2.2 \\
\hline & & & & & & & & & & & & & & \\
\hline 1 & 2 & 10 & 1.' & 1. & & 2.4 & & 2.6 & 2 & & .1 & 2.8 & 1. & 2 \\
\hline & & & 1. & & & 2.4 & & & & & & & & \\
\hline 1 & 4 & 5 & 1. & 1. & & 2.4 & 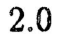 & 2.5 & 2 & & & & 1.1 & \\
\hline & & 2 & 1. & 1 & & 2. & & & & & & & .6 & \\
\hline 1 & 8 & 5 & 1.7 & 1. & & 2.4 & 2 & 2.5 & 2. & & 2.1 & & 1.2 & 1. \\
\hline & & 0 & 1.5 & & & 2.2 & .0 & 2.3 & 2.0 & 2.4 & .0 & 2.8 & .8 & 1.5 \\
\hline 5 & 5 & 20 & 1.6 & $1 . ?$ & & 2.4 & .0 & 2.7 & 2.1 & 2.6 & 2.2 & 2.8 & 1.3 & 1.9 \\
\hline & & & 1.2 & 1. & & & 6 & 2.7 & & & & 1 & .8 & 1. \\
\hline 15 & 15 & 2 & 1.9 & 2. & & 2.4 & 2.3 & 2.6 & & & 3 & 6 & 1.7 & 2 \\
\hline & & & 1. & 1 & & 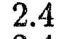 & 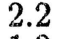 & 2. & & 2 & 2.4 & 2 & 5 & 1 \\
\hline & & & 1.5 & A. & & 2.4 & 1.9 & 2. & & 2 & & 2 & 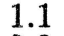 & 1 \\
\hline 30 & 30 & 20 & 2.1 & 2. & & & 2 & $?$ & & 2. & & & 20 & 2 \\
\hline & & & 1. & 0 & & 2. & 2. & 2. & & 2 & 2 & 2 & & 2.0 \\
\hline & & & 1 & & & 2.4 & 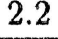 & 2.6 & & 2. & 2.4 & 2. & 1.5 & 1.8 \\
\hline 5 & 10 & 20 & 1. & 1.8 & & 2.4 & 2.2 & 2.6 & & 2 & 2. & 2 & 1. & \\
\hline & & & & & & & & & & & & & & \\
\hline 15 & 30 & & 2. & 2. & & & & 2 & & & & 2 & 1. & \\
\hline & & & 1.8 & 1. & & 2 & 2 & 2 . & 2 & & & 2 & 1. & 1.9 \\
\hline & & & & & & & & & & & & & & 1.7 \\
\hline 30 & 60 & 10 & 2. & 2. & & 2. & 0 & 2. & 2 & 2. & 2. & 2.5 & 1.9 & 2.0 \\
\hline & & & & & & 2.4 & & 2. & & 2. & 2 & 2. & 1.6 & \\
\hline
\end{tabular}

$\mathrm{L}$ and $\mathrm{U}$ stand for Lower and Upper noncoverage rates, respectively. and corr. for the continuity correction.

Results, presented in Table 1 for $\psi=1.0$, show that the original exact method, using (4) and (5), were extremely conservative, and the Sato's method, using (12) with the continuity correction, gave the almost same percentages. The mid-P method, the lnmethod, using (8) or (10), and the Sato's method without the correction gave the very accurate and similar lower and upper percentages in all designs, though the ln-method were slightly conservative in the lower tail when $n=1, m=8$, and $K=25$.

Results for $\psi=3.5$ (Table 2) show that the mid-P method were the most accurate and gave the nearest percentages to the nominal size $2.5 \%$. For the mid-P method, none out of 46 lower and upper percentages fell outside the interval $(2,3)$, while 7 and 5 out of 46 fell outside that interval for the ln-method using $V_{c}$ and $V_{R}$, respectively, and most of lower percentages were smaller than the upper percentage. For the original exact method, results show the almost symmetric but conservative. For the Sato's method, the continuity correction gave conservative results and more asymmetry than the exact method, while only 4 out of 46 lower and upper percentages fell outside the interval $(2,3)$ 


\section{Table 3}

Percentage of trials in which $\psi$ fell bellow the lower or above the upper bound of the $95 \%$ confidence interval, $\psi=6.5$.

\begin{tabular}{|c|c|c|c|c|c|c|c|c|c|c|c|c|c|c|}
\hline \multirow{4}{*}{$n$} & \multirow{4}{*}{$m$} & \multirow{4}{*}{$K$} & \multirow{2}{*}{\multicolumn{4}{|c|}{ Exact method }} & \multirow{2}{*}{\multicolumn{4}{|c|}{ ln-method }} & \multicolumn{4}{|c|}{ Sato } \\
\hline & & & & & & & & & & & \multirow{2}{*}{\multicolumn{2}{|c|}{$\begin{array}{c}\text { Without } \\
\text { corr. }\end{array}$}} & \multirow{2}{*}{\multicolumn{2}{|c|}{$\begin{array}{l}\text { With } \\
\text { corr. }\end{array}$}} \\
\hline & & & \multicolumn{2}{|c|}{ Original } & \multicolumn{2}{|c|}{ mid-P } & \multicolumn{2}{|c|}{$\hat{\psi}_{c}, V_{c}$} & \multicolumn{2}{|c|}{$\hat{\psi}_{M H}, V_{R}$} & & & & \\
\hline & & & $\mathrm{L}$ & $\bar{U}$ & $\mathrm{~L}$ & $\mathrm{U}$ & $\mathrm{L}$ & $\bar{U}$ & $\mathrm{~L}$ & $\mathrm{U}$ & $\mathrm{L}$ & $\mathrm{U}$ & $\mathrm{L}$ & $\bar{U}$ \\
\hline 1 & 1 & 200 & 1.6 & 2.0 & 2.3 & 2.4 & 1.5 & 2.9 & 1.5 & 2.9 & 1.7 & 3.2 & 1.1 & 2.4 \\
\hline & & 100 & 1.2 & 1.7 & 2.1 & 2.3 & .4 & 3.0 & .4 & 3.0 & 1.4 & 3.2 & .6 & 2.2 \\
\hline \multirow[t]{2}{*}{1} & 2 & 100 & 1.5 & 1.9 & 2.3 & 2.5 & 1.5 & 2.9 & 1.6 & 2.9 & 1.7 & 3.2 & .9 & 2.1 \\
\hline & & 50 & 1.2 & 1.7 & 2.1 & 2.4 & .4 & 2.9 & .5 & 3.0 & 1.4 & 3.4 & .4 & 2.0 \\
\hline \multirow[t]{2}{*}{1} & 4 & 50 & 1.5 & 1.7 & 2.4 & 2.4 & 1.7 & 2.7 & 1.7 & 2.7 & 1.7 & 3.1 & .7 & 1.8 \\
\hline & & 25 & .8 & 1.4 & 1.8 & 2.2 & .3 & 2.6 & .3 & 2.7 & 1.1 & 3.4 & .1 & 1.5 \\
\hline \multirow[t]{2}{*}{1} & 8 & 50 & 1.6 & 1.7 & 2.4 & 2.4 & 2.1 & 2.5 & 2.0 & 2.6 & 1.9 & 2.9 & .8 & 1.6 \\
\hline & & 05 & 1.4 & 1.5 & 2.2 & 2.3 & 1.6 & 2.5 & 1.5 & 2.6 & 1.6 & 3.2 & .4 & 1.5 \\
\hline \multirow[t]{2}{*}{5} & 5 & 20 & 1.4 & 1.6 & 2.3 & 2.4 & 1.7 & 2.8 & 1.7 & 2.7 & 1.9 & 3.0 & .8 & 1.8 \\
\hline & & 10 & .8 & 1.4 & 1.7 & 2.3 & .6 & 2.8 & .7 & 2.7 & 1.3 & 3.2 & .2 & 1.6 \\
\hline \multirow[t]{3}{*}{15} & 15 & 20 & 1.9 & 1.9 & 2.4 & 2.4 & 2.2 & 2.6 & 2.3 & 2.6 & 2.3 & 2.6 & 1.5 & 1.9 \\
\hline & & 10 & 1.7 & 1.7 & 2.4 & 2.4 & 2.0 & 2.7 & 2.1 & 2.6 & 2.2 & 2.7 & 1.2 & 1.8 \\
\hline & & 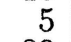 & 1.3 & 1.5 & 2.2 & 2.3 & 1.5 & 2.8 & 1.6 & 2.7 & 1.9 & 3.0 & .7 & 1.6 \\
\hline \multirow[t]{3}{*}{30} & 30 & 20 & 2.1 & 2.2 & 2.5 & 2.6 & 2.3 & 2.7 & 2.3 & 2.7 & 2.3 & 2.7 & 1.8 & 2.1 \\
\hline & & 10 & 2.0 & 1.9 & 2.6 & 2. & 2.3 & 2.7 & 2.4 & 2.6 & 2.5 & 2.7 & 1.6 & 1.9 \\
\hline & & 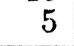 & 1.6 & 1.7 & 2.3 & 2.3 & 2.0 & 2.7 & 2.0 & 2.5 & 2.2 & 2.7 & 1.1 & 1.7 \\
\hline \multirow[t]{2}{*}{5} & 10 & 20 & 1.7 & 1.8 & 2.4 & 2.4 & 2.1 & 2.7 & 2.1 & 2.7 & 2.2 & 2.9 & 1.1 & 1.8 \\
\hline & & 10 & 1.3 & 1.6 & & 2. & 1.6 & 2.7 & 1.8 & 2.6 & 2.1 & 2.9 & .6 & 1.5 \\
\hline \multirow{3}{*}{15} & 30 & 20 & 2.0 & 2.1 & 2.5 & 2.5 & 2.4 & 2.6 & 2.4 & 2.6 & 2.5 & 2.6 & 1.7 & 2.0 \\
\hline & & 10 & 1.8 & 1.8 & 2. & 2. & 2.2 & 2.6 & 2.3 & 2.5 & 2.4 & 2.6 & 1.4 & 1.6 \\
\hline & & $r$ & 1.5 & 1.7 & 2.3 & 2.4 & 1.9 & 2.8 & 2.0 & 2.7 & 2.2 & 2.9 & 1.0 & 1.6 \\
\hline \multirow[t]{2}{*}{30} & 60 & 10 & 2.1 & 2.0 & 2.5 & 2. & 2.4 & 2.6 & 2.4 & 2.5 & 2. & 2.6 & 1.7 & 1.9 \\
\hline & & 5 & 1.8 & 1.9 & 2. & 2. & 2.1 & 2.7 & 2.2 & 2.6 & 2.3 & 2.7 & 1.3 & 1.8 \\
\hline
\end{tabular}

$\mathrm{L}$ and $\mathrm{U}$ stand for Lower and Upper noncoverage rates, respectively.

and corr. for the continuity correction.

without the correction. However, most of lower percentages were smaller than the upper percentage for the Sato's method with or without the correction. Results for $\psi=6.5$ (Table 3) show that the original exact method gave conservative percentages, especially lower percentages in matched designs, and that the mid-P method gave the most accurate and nearest percentages to the nominal size $2.5 \%$, and only 2 out of 46 lower and upper percentages fell outside the interval $(2,3)$. For the ln-method, both using $V_{c}$ or $V_{R}, 7$ out of 8 lower percentages fell outside the interval $(2,3)$ and were quite conservative when the total sample size was small in matched designs, but 3 out of 8 fell outside that interval, respectively, in balanced designs, and only 2 and 1 out of 7 fell outside that interval for using $V_{c}$ and $V_{R}$, respectively, in inbalanced designs, while none out of 46 upper percentages fell outside that interval. The Sato's method with the continuity correction gave more conservative lower percentages than those of the original exact method, while upper percentages were conservative but similar to those of the original exact method in all designs. For the Sato's method without the correction, upper percentages were liberal 
in all designs but near to the nominal size $2.5 \%$ in blanced and inbalanced designs, while lower percentages in matched designs were conservative but 3 out of 8 and none out of 7 lower percentages fell outside the interval $(2,3)$ in balanced and inbalanced designs, respectively. In all designs, the Sato's method without the correction gave the nearest two-tailed percentages to the nominal size $5 \%$, regardless of the sample size, the number of strata, and the true common odds ratio.

\section{Discussion}

For computing the confidence limits of the common odds ratio in a series of $2 \times 2$ tables, We compared respective results of the original exact method, the mid-P modification method, the ln-method using the conditional maximum likelihood estimate $\hat{\psi}_{c}$ and its variance $V_{c}$, or using the Mantel-Haenszel estimate $\hat{\psi}_{M H}$ and its asymptotic variance $V_{R}$ proposed by Robins et al (1986), and the Sato's method (1990) with and without the continuity correction, through Monte Carlo experiments.

Two methods for the ln-method, using $\hat{\psi}_{c}$ and $V_{c}$, and using $\hat{\psi}_{M H}$ and $V_{R}$, gave the similar results. In general, all of the approximate methods gave the accurate and symmetric lower and upper percentages when the true common odds ratio was unity, while upper percentages became liberal but lower percentages conservative, reversely, as the true common odds ratio increased, especially in matched designs. Though the Sato's method without the continuity correction gave the nearest two-tailed percentages to the nominal size $5 \%$ in all designs, asymmetric results were found in matched designs as well.

In all designs including both sparse-data settings and large-stratum settings, the mid-P exact method gave the most accurate and nearest lower and upper percentages to the nominal size $2.5 \%$, regardless of the true common odds ratio. According to the results, we should recommend the mid-P exact method to construct the confidence interval for the common odds ratio. However, for the mid-P method, 5 out of 138 percentages exceeded to the nominal size $2.5 \%$. Therefore, if it is thought that the actual percentage must be smaller than the nominal size, the original exact method is favor as well as the Sato's method with the continuity correction that gave conservative results in all designs.

\section{References}

Breslow, N.E. (1981). Odds ratio estimators when the data are sparse. Biometrika 68, 73-84.

Gart, J.J. (1970). Point and interval estimation of the common odds ratio in the combination of $2 \times 2$ tables with fixed marginals. Biometrika 57, 471-475.

Hauck, W.W. (1979). The large sample variance of the Mantel-Haenszel estimator of a common odds ratio. Biometrics 35, 817-819.

Lancaster, H.O. (1961). Significance tests in discrete distributions. Journal of the American Statistical Association 56, 223-234.

Mantel, N. and Haenszel, W. (1959). Statistical aspects of the analysis of data from 
retrospective studies of disease. Journal of the National Cancer Institute 22, 719748.

Mehta, C.R., Patel, N.R., and Gray, R. (1985). Computing an exact confidence interval for the common odds ratio in several $2 \times 2$ contingency tables. Journal of the American Statistical Association 80, 969-973.

Robins, J.M., Breslow,N.E., and Greenland, S. (1986). Estimators of the Mantel-Haenszel variance consistent in both sparse data and large-strata limiting model. Biometrics 42, 311-323.

Rothman, K.J. (1986). Modern Epidemiology, Little, Brown and Company, Boston.

Sato, T. (1990). Confidence limits for the common odds ratio based on the asymptotic distribution of the Mantel-Haenszel estimator. Biometrics 46, 71-80.

Takagi, H. (1990). A simple recursive algorithm for the exact confidence limits for the common odds ratio in a series of $2 \times 2$ tables. Research Memo., No.385, the Institute of Statistical Mathematics.

Vollset, S.E. and Hirji, K.F. (1991). A microcomputer program for exact and asymptotic analysis of several $2 \times 2$ tables., Epidemiology 2, 217-220.

Zelen, M. (1971). The analysis of several $2 \times 2$ contingency tables. Biometrika 58, 129-137.

[Received June, 1991] 